\title{
The Coupled Atomistic/Discrete-Dislocation Method in 3d Part III: Dynamics of hybrid dislocations
}

\author{
Jaehyun Cho ${ }^{\mathrm{a}}$, Jean-François Molinari ${ }^{\mathrm{a}}$, William Curtin ${ }^{\mathrm{b}}$, Guillaume Anciaux ${ }^{\mathrm{a}, *}$ \\ ${ }^{a}$ Ecole Polytechnique Fédérale de Lausanne (EPFL) \\ Faculté ENAC-IIC, LSMS (http://lsms.epfl.ch/) \\ CH-1015 Lausanne, Switzerland \\ ${ }^{b}$ Ecole Polytechnique Fédérale de Lausanne (EPFL) \\ Faculté STI, LAMMM (http://lammm.epfl.ch/) \\ CH-1015 Lausanne, Switzerland
}

\begin{abstract}
In two companion papers, the CADD-3d method for coupling atomistic and discrete dislocations was described and validated for (quasi-)static equilibrium. Here, CADD-3d is validated further for the time evolution of straight and curved hybrid dislocations, i.e. dislocations that span both atomistic and continuum domains simultaneously. Studies show physically sensible results when using precise calibration of the discrete dislocation mobility and atomistic core structures, and the sensitivity of results when intentional deviations away from the precise calibrations are used. CADD-3d is then used to model the evolution of dislocation loops from atomistic Frank-Read sources under sustained load. The predicted nucleation and expansion of multiple loops is in excellent agreement with fully atomistic simulations at small scales. CADD$3 \mathrm{~d}$ is then applied to much larger scales that capture both the nucleation of loops at the atomic scale and the evolution of a dislocation loop pile-ups at the micron scale, with full interactions among all dislocations in the system during the entire time evolution. Collectively, the results here serve to demonstrate the accuracy, robustness, and power of CADD-3d, and point toward its future application in many complex dislocation problems requiring both atomistic resolution and large-scale dislocation evolution.
\end{abstract}

Keywords: CADD-3d, CADD, Multiscale modeling, hybrid dislocation, Molecular dynamic, Discrete dislocation dynamics

\section{Introduction}

The deformation and strength of plastically-deforming crystalline materials is controlled by dislocation line defects, which nucleate, expand, glide, and are pinned during deformation. The collective evolution of the dislocation network gives rise to the macroscopic plastic flow behavior of yielding and strain hardening [28]. Dislocations are atomistic defects, and accurate description of the highly-distorted core of the dislocation requires an atomistic description achieved through molecular dynamics (MD) simulations. At the same time, the long-range interactions among dislocations are elastic in nature, and dislocation networks form over the scale of microns. These features are beyond the computational ability of MD but can be captured using the larger-scale discrete dislocation dynamics (DDD) method that employs underlying atomistic information into elastic energies, core energies, and dislocation mobility.

MD represents every single atom, interacting via semi-empirical interatomic potentials and capturing the detailed vibration and deformation of all atoms. A single dislocation is then represented by a particular configuration of (many) atoms around the termination of a slip along a slip plane that form the dislocation core, and associated longer-range elastic distortions of the crystalline material. The DDD method represents the dislocation line only as a continuum line defect embedded in a surrounding elastic medium [6]. The

\footnotetext{
*Corresponding author: guillaume.anciaux@epfl.ch
} 
line defect is discretized into nodes connected by segments whose evolution is achieved by computing the total force (due to applied fields, elastic interactions with other segments, and self-forces) on each segment and then applying mobility laws to move the nodes/segments [7]. The computational cost of DDD is thus significantly lower than MD, allowing the study of plasticity problems with many dislocations in micrometersized crystals [2]. However, the absence of atomistic detail precludes the use of DDD from accurately study dislocation nucleations and/or dislocation interactions with other atomistic defects (such as solutes, grain boundaries, voids, precipitates, and cracks).

The gap between MD and DDD descriptions motivates the development of concurrent multiscale methods that handle both scales simultaneously. However, the major challenge has been the handling of dislocation lines that span the MD and DDD domains simultaneously, what we call hybrid dislocations. Multiscale methods to date have thus been limited to coupling of atomistics to a hyper-elastic continuum domain containing no dislocations ([24]) or to plane-strain problems where individual dislocations reside in only one domain or the other at any instant, with algorithms for passing dislocations across the atom/continuum interface [25]. In a companion paper, Paper I [1], we have presented a new CADD-3d method that handles the challenging problem of hybrid dislocations. In a second paper, Paper II [14], key features of CADD-3d were validated in a static equilibrium context, demonstrating that CADD-3d could achieve excellent detailed agreement with fully atomistic simulations of the same problem.

In the present paper, CADD-3d is evaluated for the dynamical evolution of various hybrid dislocations. We use the algorithms detailed in Paper I plus the simplification of using DDD elastic fields in infinite space to avoid the need for FE solutions of the DDD problem as discussed in Paper II. These algorithmic details are not repeated here; this paper focuses on further validation and results.

In Section 2, the propagation of hybrid straight dislocations is studied to demonstrate the robustness of the method when the DDD mobility law and so-called core-template that connects the DDD line defect to the atomistic dislocation defect, are accurately calibrated to atomistic properties. In section 3 we present a strategy to pass the initially purely atomistic dislocations through the coupling interface. Then, in Section 4 we consider the dynamic evolution of a hybrid dislocation loop expanding simultaneously in both atomistic and DDD domains under an applied shear load, and in the presence of additional dislocations, and we study the shrinking and eventual annihilation of a loop under zero applied load. These highlight the ability of CADD-3d to capture highly curved dislocations and the motion of dislocations into and out of the atomistic domain, when using the elasticity method described in Paper II. Finally, in Section 5, CADD-3d is used to model the evolution of dislocation loops from an initial Frank-Read source, showing bowing-out, pinching off to form discrete loops, expansion and passing of hybrid dislocation loops into fully-DDD loops, and further expansion of the DDD loops over large scales, with full elastic coupling of all dislocations in the system. With these validations and demonstrations, CADD-3d is shown to be suitable for application to complex dislocation plasticity problems that require full atomistic resolution in local domains plus dislocation network evolution at scales unobtainable in atomistic simulations.

\section{Hybrid Straight dislocations}

The first dynamical evaluation is the assessment of the steady-state motion of a straight hybrid dislocation under a uniform applied load. In a perfect method, the dislocation should remain straight and glide at a steady average velocity. This simple problem thus allows us to study deviations from the perfect behavior due to deviations in the DDD calibration and/or structure of the core template used to couple atomistic and continuum parts of the hybrid dislocation.

The study is carried out for a range of different dislocation character angles. Figure 1 illustrates the general case for a mixed dislocation with character angle $\vartheta$. A straight hybrid dislocation is modeled as periodic along the line direction with a small length of $15|\boldsymbol{b}|$ represented atomistically and a much larger length $(1670|\boldsymbol{b}|)$ represented by DDD. The atomistic domain $\Omega_{\mathrm{A}}$ has dimensions $15|\boldsymbol{b}|$ along the line direction, $110|\boldsymbol{b}|$ along the glide direction, and $15|\boldsymbol{b}|$ in the direction normal to the glide plane. The surrounding pad region $\Omega_{\mathrm{P}}$ has a thickness of $7 \AA$, and results do not depend on pad thickness for larger values. The transmission nodes, as defined in Paper II, are the first red nodes indicated in Figure 1b. We use the Mendelev interatomic potential 
for Aluminum [18] and the simulation is performed at a low temperature $\sim 1$ Kelvin. Character angles are those for which we previously computed core templates and atomistic steady state mobilities [10, 11], namely 90 (edge), 60, 49.107, 40.893, 30, 19.107 and 0 (screw) degrees.

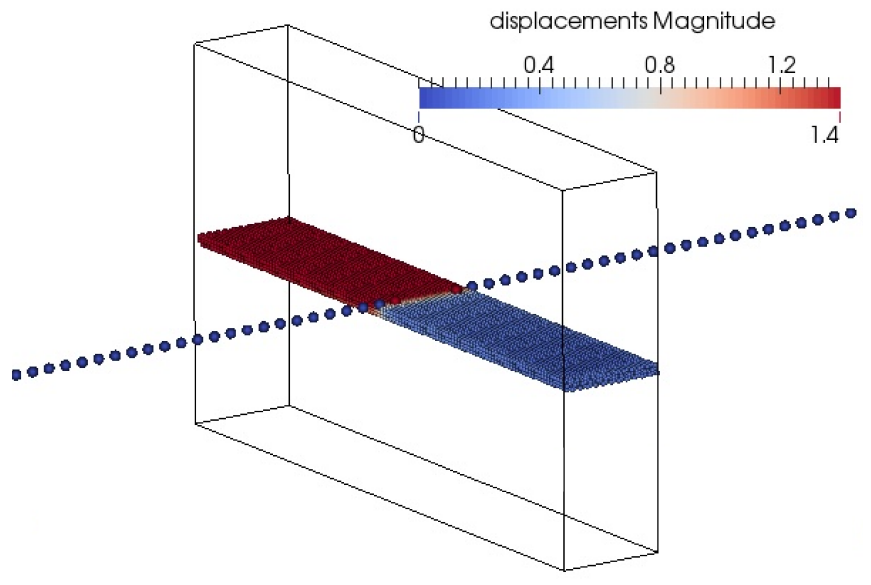

(a)

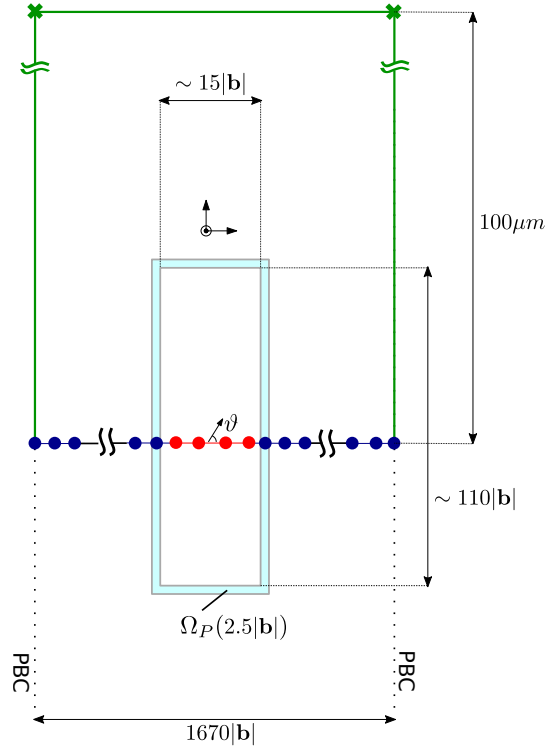

(b)

Figure 1: (a) Hybrid straight dislocation with a central 3d MD domain and a surrounding 3d DDD domain. Only atoms around the bottom slip plane are shown, and are colored by the magnitude of displacement relative to undeformed perfect lattice positions. DDD nodal points are shown as large blue spheres. The black frame identifies the entire MD region and the pad region $\Omega_{\mathrm{A}} \cup \Omega_{\mathrm{P}}$. (b) Top view schematic of the hybrid dislocation slip plane, also showing the additional dislocation segments (green lines) necessary to form the closed loop needed to compute displacement fields using Barnett's solution [3, 4, 12]. The nodes in the MD region (red disks) are positioned thanks to the dislocation detection.

A homogeneous pure shear stress is applied at infinity, with shearing direction aligned with the Burgers vector $\boldsymbol{b}$. As described in Paper II, the DDD dislocation evolves due to the uniform applied shear plus the elastic forces among all the dislocation segments (including segments detected in the atomistic domain). The atomistic motion is controlled by the imposed displacements in the pad region, due to the shear and the - fields of the entire dislocation line plus the core template correction near the dislocation core. Specifically, the displacements applied to the atomistic domain are

$$
\boldsymbol{u}_{a}=\hat{\boldsymbol{u}}\left(\boldsymbol{x}_{a}\right)+\tilde{\boldsymbol{u}}\left(\boldsymbol{x}_{a}, \gamma^{\mathrm{c}}, \gamma^{\mathrm{v}}\right)+\Delta \tilde{\boldsymbol{u}}^{\mathrm{corr}}\left(\boldsymbol{x}_{a}\right) \quad \forall \boldsymbol{x}_{a} \in \Omega_{\mathrm{P}},
$$

where $\hat{\boldsymbol{u}}$ and $\tilde{\boldsymbol{u}}$ are the $\hat{\bullet}$ and $\tilde{\bullet}$ contributions to the displacement field and $\Delta \tilde{\boldsymbol{u}}^{\text {corr }}$ is the core template correction applied within a radius $R^{\text {core }}=10|\boldsymbol{b}|$ from the dislocation line. The displacement field is simply

$$
\hat{\boldsymbol{u}}_{\mathrm{x}}=\frac{\hat{\boldsymbol{\sigma}}_{\mathrm{xy}}}{\mu} y, \quad \hat{\boldsymbol{u}}_{\mathrm{y}}=0, \quad \hat{\boldsymbol{u}}_{\mathrm{z}}=\frac{\hat{\boldsymbol{\sigma}}_{\mathrm{yz}}}{\mu} y
$$

where $\mu$ is the shear modulus ${ }^{1}$. The displacement field $\tilde{\boldsymbol{u}}$ is computed using the Barnett solution [3, 4, 12], which requires a closed dislocation loop. The loop is thus closed by adding fixed/immobile segments very far $(100 \mu \mathrm{m})$ from the atomistic domain to create a very large loop as indicated by the green line in Figure 1 . To avoid spurious waves at the onset of the simulation, the initial displacement field is imposed on all atoms in $\Omega_{\mathrm{A}}$ at $\mathrm{t}=0$. Recall from Paper II that this approach to computing the displacement fields is suitable when only dislocations exist in the problem, such that the entire dislocation network, including the atomistic region, is known (with the dislocations in the atomistic region identified using the detection algorithm).

The time integration is performed explicitly in the form of a predictor-corrector scheme (steps (1) , (4) in Algorithm 1 of Paper I [1]). In order to save computational cost, we can use sub-cycling of the DDD

\footnotetext{
${ }^{1}$ The low level of anisotropy of aluminum allows to use a single shear modulus independent of the character angle.
} 

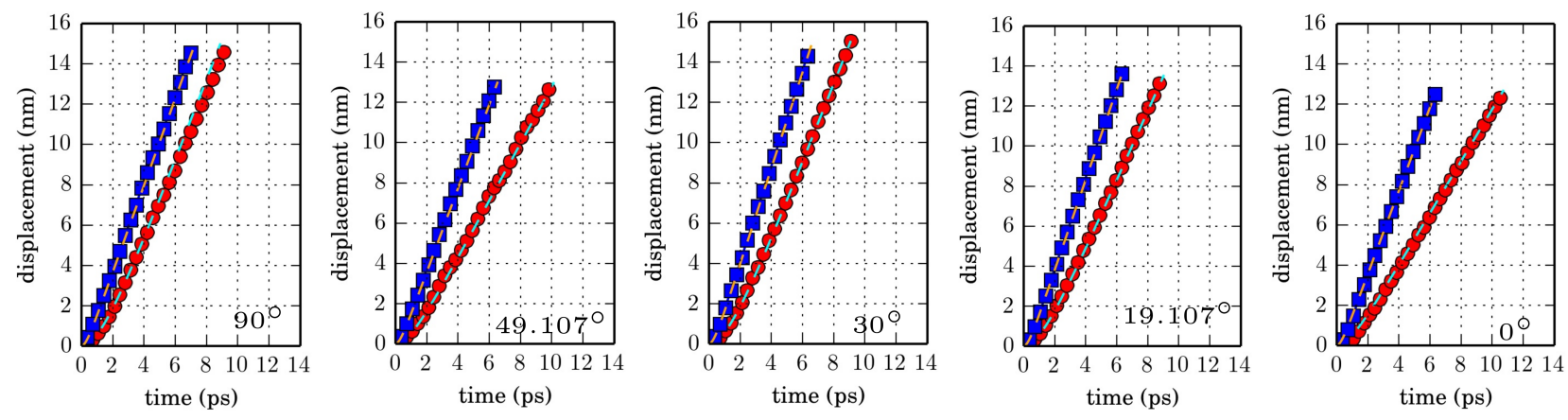

Figure 2: Displacement of a straight hybrid dislocation versus time with a shear stress applied at $\mathrm{t}=0$. Results are shown for 5 selected character angles and two shear stresses (100MPa and $500 \mathrm{MPa})$. Circles indicate average of atomistic portion of the hybrid dislocation and dashed lines indicate average of the DDD portion of the hybrid dislocation.

sub-problem, with a DDD update time that is much longer than the MD time-step $\Delta t_{M D}$. Here, we update the DDD problem at time increments of $\Delta t_{\text {update }}=50 \Delta t_{M D}$. We show below that this choice is a good compromise between accuracy and computational cost.

Under the applied shear load, the dislocation will first accelerate before stabilizing to an average steadystate velocity. Mobility laws relating the resolved shear stress $\sigma_{\text {res }}$ and velocity $v$ describe steady-state motion $[11,20]$ with the angle dependent coefficient $\mathcal{D}_{\vartheta}$ when the velocity is much smaller than the shear wave speed and when $\sigma_{\text {res }} \geq \sigma_{\text {Peierls }}$. To include inertia that is observed in MD simulations (see $[5,21,22,13,19]$ ) leading to a transient behavior prior to reaching steady-state, the mobility relation is modified as

$$
|\boldsymbol{b}|\left(\sigma_{\text {res }}-\sigma_{\text {Peierls }}\right)=\mathcal{D}_{\vartheta} v+m^{d} \dot{v}
$$

where the effective mass density $m^{d}$ is determined from fully-atomistic studies. Although $m^{d}$ could depend on the character angle, here we use a character-independent value of $m^{d}=0.1[\mathrm{Kg} / \mathrm{m}]$ in all simulations. For more details, the reader is referred to the appendix of [1].

Figure 2 shows the time evolution of the atomistic and DDD portions of the hybrid dislocation during glide, for a range of character angles and two applied shear stresses (100 MPa, $500 \mathrm{MPa})$. These results are obtained using the atomistically-calibrated DDD mobilities and accurate atomistic core templates. The dislocations remain very straight, thus revealing that no significant spurious forces are created by the CADD-3d coupling. Figure 3a shows a typical example of the $30^{\circ}$ hybrid dislocation after $5.5 \mathrm{ps}$. The atomistic dislocation is not perfectly straight - there is a very slight curvature and a lagging of the atomistic dislocation by $\approx 1|\boldsymbol{b}|$; this can be attributed to very slight errors in the DDD calibration. The CADD-3d method is thus validated for the simplest dynamic dislocation problem.

The use of intentionally incorrect or less accurate calibrations leads to demonstrable errors. We demonstrate the sensitivity of the results to the calibration inputs for a $30^{\circ}$ dislocation under a shear stress of $100 \mathrm{MPa}$. We vary one calibration input at a time, with all other parameters held at the accurate calibrated values.

First, we use a core template with a small cut-off radius $R^{\text {core }}=|\boldsymbol{b}|$ (versus the $10|\boldsymbol{b}|$ radius used above). Figure $3 \mathrm{~b}$ shows snapshots of the hybrid dislocation at 0ps and 5.5ps. Comparison with the reference calibration indicates that the small core template exerts some spurious drag force that slows down the motion of the hybrid dislocation. The atomistic configuration near the core template is distorted by the incorrect template (with a too small cut-off radius), generating the spurious forces. Figure 4 shows the position of the center of the atomistic portion of the dislocation as a function of time, quantifying the deviation from the reference calculation. The final velocity is similar to the reference case but the dislocation lags behind the well-calibrated reference case.

Second, we change the mobility damping parameter by factors of $\frac{1}{2}$ and 2 , which leads to a higher or lower steady-state velocity, respectively. The resulting hybrid dislocation motion when the damping is reduced by $1 / 2$ is shown in Figure 3c, where it is observed that the continuum portion of the dislocation is moving 
ahead of the atomistic portion. This generates additional forces on the atomistic portion, and ultimately a steady-state velocity is achieved but with the hybrid dislocation being far from straight. When the damping parameter is increased to twice the reference value, the continuum portion lags behind the atomistic portion, which exerts a drag on the atomistic domain. The position of the atomistic dislocation is shown in Figure 4 for both cases as a function of time.

Third, we neglect inertia of the continuum dislocation by setting the effective mass $m^{d}$ to zero. This reduces the duration of the acceleration phase from $\sim 2 p s$ to $\sim 0.5 p s$. The DDD portion pulls the entire dislocation line forward until a steady-state velocity that matches the reference case is achieved (after $\sim 0.5 p s$ ). Whereas the dislocation advanced ahead of the reference case because of the acceleration phase, it later moves at the correct steady-state velocity, so that the error due to a wrong effective mass remains bounded.

Finally, we use a large continuum update time step $\left(t_{\text {update }}=200 \Delta t\right)$, which again shows deviations in dislocation position versus time when compared to the reference state. With such a large time step, the distance a dislocation glides every update step at a steady-state velocity of $1.5 \mathrm{~nm} / \mathrm{ps}$ is $3 \AA$, which is comparable to the Burgers vector length, as compared to the distance of $0.75 \AA$ for an update frequency of $50 \Delta t$.

The above sensitivity studies demonstrate that all of the calibrations involved in CADD-3d are necessary to achieve accurate atomistic/continuum dislocation coupling. Inaccurate calibrations are immediately manifested in distortions of the dislocation line from the straight configuration. Since accurate calibration of mobility laws and the core template are achieved with very accessible MD simulations, there is no significant barrier to calibrating CADD-3d to achieve excellent results as compared to fully atomistic simulations.

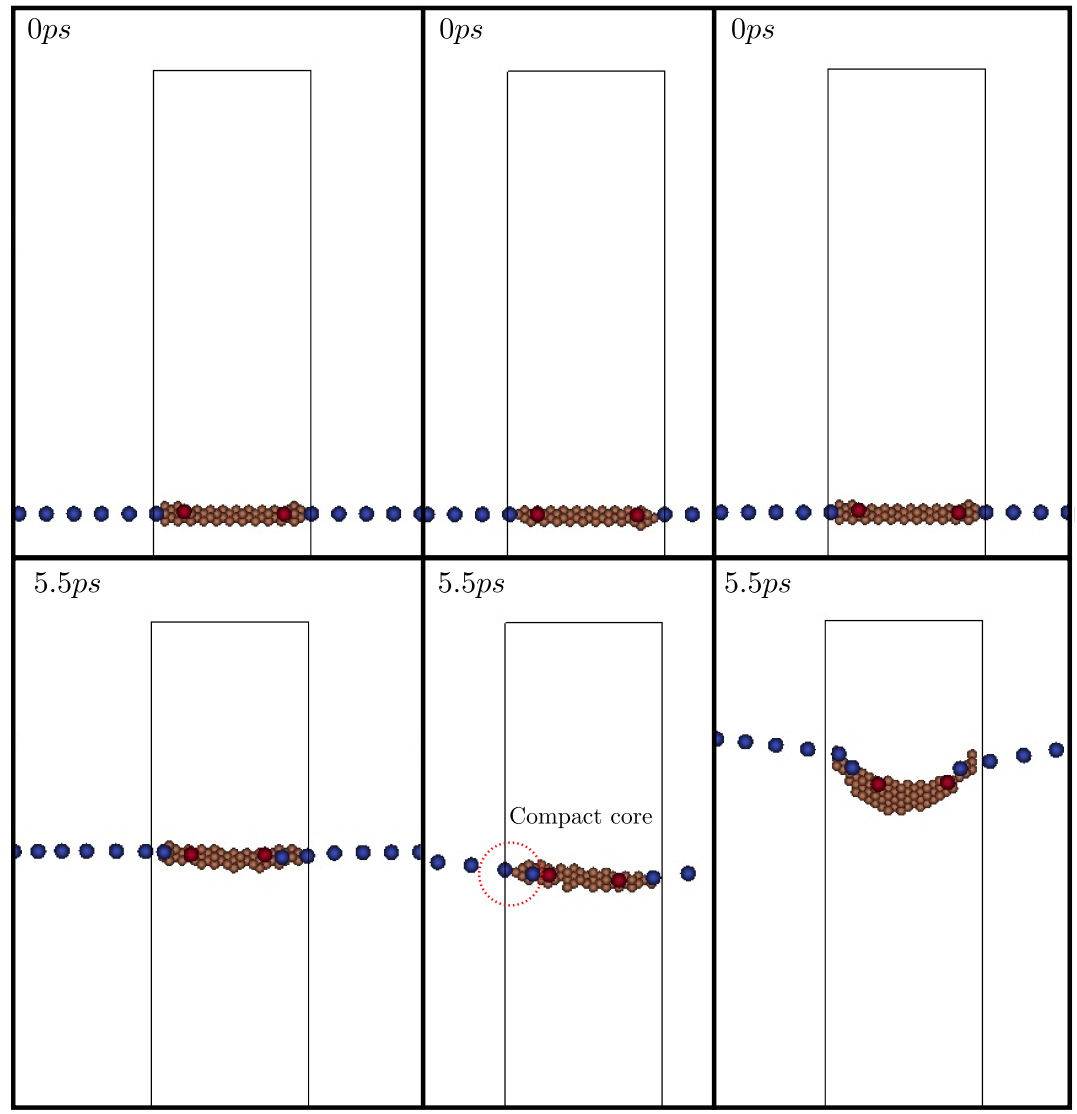

(a) Calibrated

(b) Case 1

(c) Case 2

Figure 3: Snapshots of the dynamics of a mixed $30^{\circ}$ hybrid dislocation under a $100 \mathrm{MPa}$ applied load, top view of the slip plane where the DDD nodes and the atoms violating centro-symmetry are plotted. (a) Simulation result obtained in the case where the coupling parameters are adequately chosen, (b) obtained with a small cut-off radius $R^{\text {core }}=|\boldsymbol{b}|$ for the core correction, (c) and with $B_{\vartheta}:=\frac{1}{2} B_{\vartheta}$. 


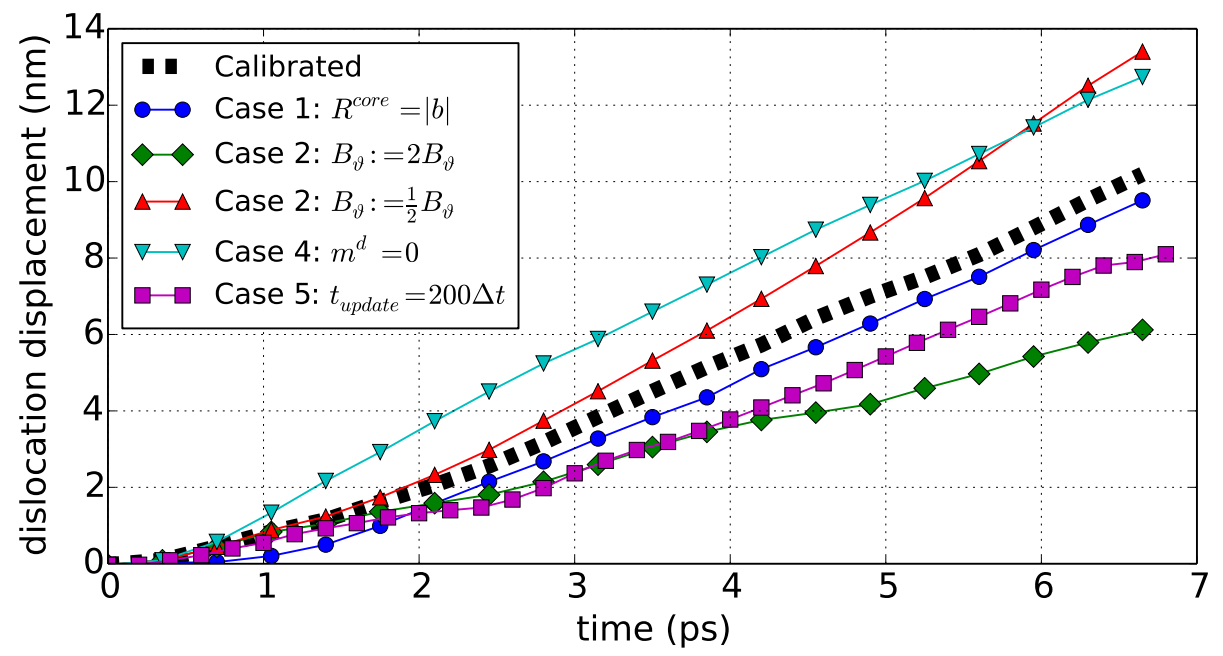

Figure 4: Position of the center of the MD dislocation versus time, for a range of intentionally mis-calibrated inputs to CADD-3d and for the accurate calibration. Mis-calibrations of the core template cut-radius, damping parameter $B_{\vartheta}$, effective mass $m$, and continuum update step $t_{\text {update }}$ are shown.

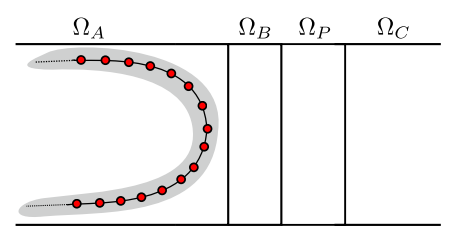

(a)

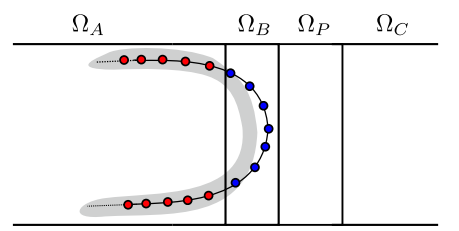

(b)

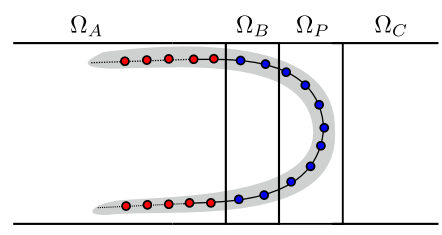

(c)

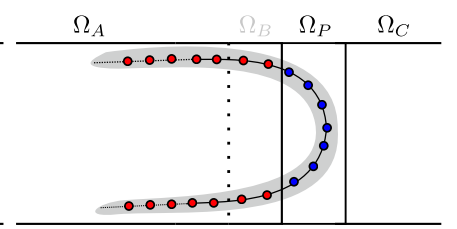

(d)

Dislocated atoms $\quad$ Slaved DD nodes $\bigcirc$ Free DD nodes

Figure 5: Illustration of the dislocation passing, including the additional buffer zone $\Omega_{B}$ where dislocation segments are set independent from the atoms. (a) A dislocation approaches the coupling region and is detected in $\Omega_{\mathrm{A}}$. (b) Dislocation nodes entering the buffer region are set free from the atomic description and now react to Peach-Koehler forces, allowing the dislocation nodes to approach the PAD region $\left(\Omega_{\mathrm{P}}\right)$. MD and DD descriptions might slightly split during this phase. However, these free nodes induce a MD template (displacement) field in $\Omega_{\mathrm{P}}$ that will pull the MD dislocation towards the continuum region. (c) Several nodes entered the pad region, and a hybrid dislocation is now formed. (d) The free nodes in the buffer region can be slaved again since a proper hybrid dislocation is formed. 


\section{Dislocation passing}

When a fully-atomistic dislocation glides toward the boundary of the atomistic domain $\Omega_{\mathrm{A}}$, the boundary conditions applied on the pad region $\Omega_{\mathrm{P}}$ introduce artificial forces. As shown in Paper II [14], such forces exist for all coupling methods including the full multiscale non-linear atomistic/continuum coupling. We also showed that the forces are larger when using the simplified method in which a fully DDD representation of the dislocation network is used to compute the pad atom displacements. Dislocations thus cannot naturally and automatically pass from a fully atomistic representation to a hybrid representation. Dewald and Curtin showed that the spurious forces can be partially mitigated using the core template in the $2 \mathrm{~d}$ setting. Using the simplified method introduced in Paper II and used in this paper, where all fields are computed via DDD on the entire dislocation network, an algorithm for passing becomes possible, as discussed below.

The algorithm for passing is illustrated in Figure 5. An initial dislocation lies inside the atomistic domain at some distance from the pad (Figure 5a). The detection algorithm is operating at all times to detect the atomistic dislocation. The DDD nodes and segments are completely slaved to the atomistic dislocation, and motion of the dislocation is due to atomic motions. After some glide, a portion of the atomistic dislocation will enter a domain $\Omega_{B}$ just inside the pad region, as indicated in Figure 5b. The nodes detected in $\Omega_{B}$ are then treated as real DDD nodes. That is, these nodes are no longer slaved to the atomistic dislocation line and are moved according to the DDD forces. During a time increment, the DDD dislocation segments in $\Omega_{B}$ and the underlying atoms move similarly but with slight differences. However, the underlying atoms are subject to boundary conditions on $\Omega_{\mathrm{P}}$ due to the DDD segments. Thus, while the underlying atoms are not directly tied to the DDD segments, they are guided to move generally with/toward the DDD segments due to the forces they experience from the pad atoms, which are controlled by the DDD segments. The DDD segments in $\Omega_{B}$ can move across the atom/continuum interface with no hinderance because they are already treated as DDD segments. Once some portion of the loop crosses into the DDD region, the entire loop becomes a true hybrid dislocation. The DDD nodes remaining in $\Omega_{B}$ should again be slaved to the atomistic dislocation in order to be a hybrid dislocation as described in paper I [1]. Turning back such nodes into slaves is not conceptually difficult, however it requires exploration of the entire dislocation network in order to distinguish previously-passed hybrid dislocations from newly approaching purely atomic dislocations. For simplicity, results presented in sections 4 and 5, leave the dislocation nodes in the buffer $\Omega_{B}$ as controlled by DDD during the entire simulation.

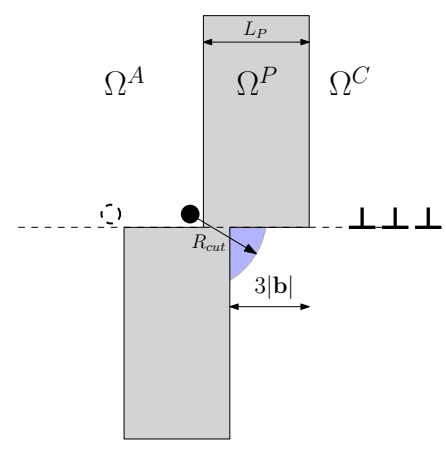

(a)

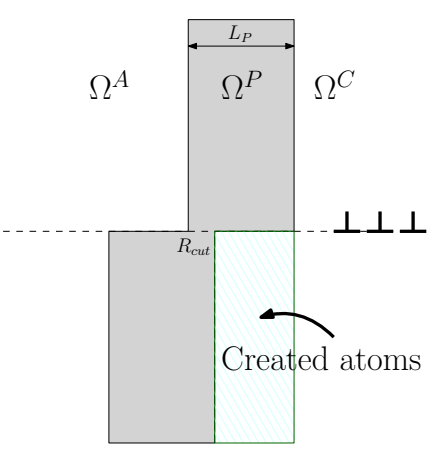

(b)

Figure 6: In (a) three dislocations have passed leaving a step of size $3|\boldsymbol{b}|$. The atom in $\Omega_{\mathrm{A}} \backslash \Omega_{\mathrm{P}}$ originally located as the dashed circle moves to the black disk. Assuming an inter-atomic distance of $r_{\mathrm{c}}$ this atom undergoes the influence of the empty region indicated in blue. (b) The region in cyan is filled with new atoms that were not existing at the onset of the simulation.

A minor issue arises when multiple dislocations pass through the coupling interface on exactly the same slip plane, as described on figure 6 . The slip displacement induces a relative shift of the pad atoms above and below the slip plane. Since atomic interactions extend over some finite distance $R_{\text {cut }}$, the shifting of the pad can cause real atoms that are next to the pad and adjacent to the slip plane to see an incomplete set of atomic neighbors (see Figure 6a, b). The response of such atoms is thus incorrect. To remedy this situation, additional pad atoms must be added to the pad domain or the pad domain must be redefined; i.e. the pad domain must be adaptive. Since the underlying crystalline reference lattice is known, and the deformation field due to the dislocation network is known, adding atoms to the pad is not conceptually difficult. We have 


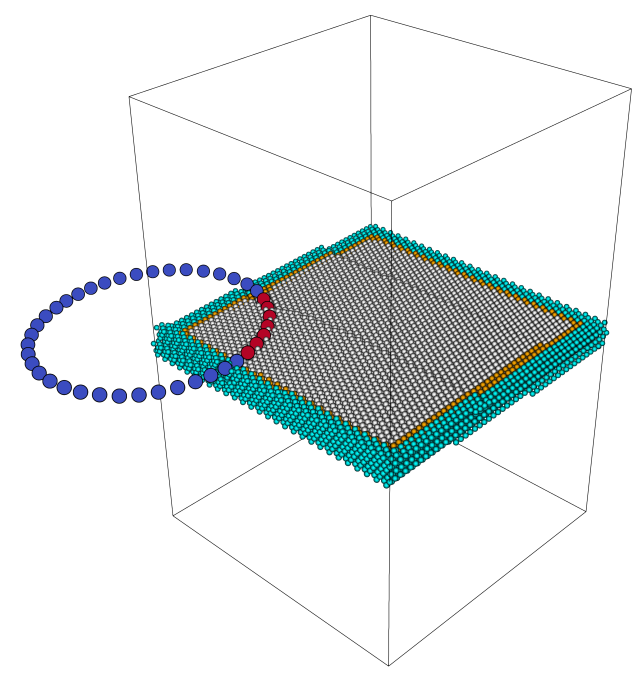

(a)

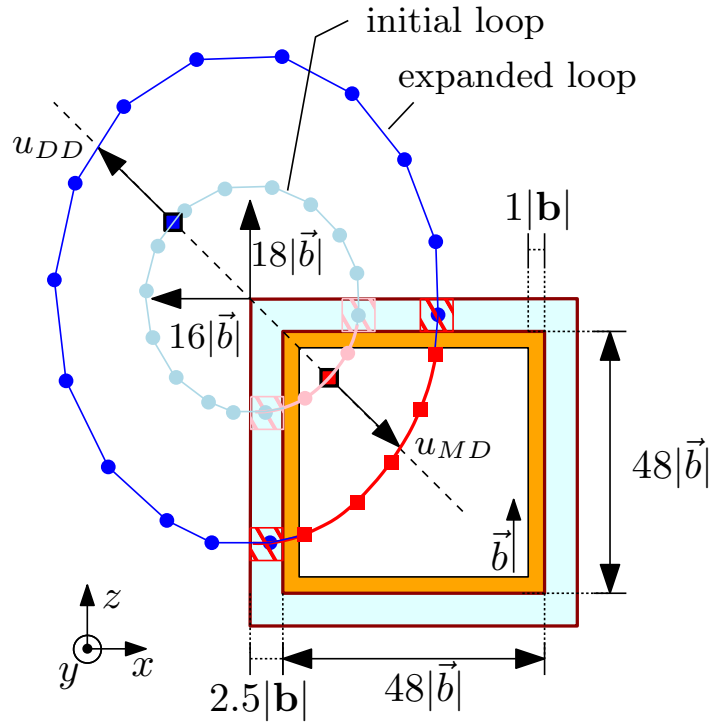

(b)

Figure 7: (a) Perspective view of the simulation box. Only the atoms around the slip plane and the DDD nodes are shown. The colors applied on the atoms highlight the pad (cyan) and buffer (orange) regions. (b) Schematic showing the hybrid dislocation from a top view of the slip plane, and how its displacement is measured in the MD and DDD regions.

developed one algorithm for cuboidal atomistic cells of single crystal in which a method usually employed for periodic cell simulations can be adapted, as described in [8].

\section{Hybrid dislocation loops}

We now consider the dynamics of curved hybrid dislocation loops. Figure 7 presents a schematic of the computational domain, consisting of a single hybrid dislocation loop with Burgers vector aligned with the $z$ axis. Initially, one quarter of the loop is atomistic and the remainder is modeled with DDD. As above, we use the Mendelev EAM potential [18] at T 1 Kelvin, and the same mobility function, interpolated between the calibrated discrete set of character angles [10,11].

There are various ways to initialize the system. The most direct approach is to insert a discrete dislocation representation into the simulation cell and use the DDD and applied shear displacement fields to initialize all atomic displacements plus the core template. During the initial stages of relaxation, the true atomistic core is rapidly formed.

As in Paper II, this problem also involves evolving dislocation character angles at the atomistic/continuum interface. The evolving character angle arises due to changes in shape of the dislocation and due to changes in the specific boundary through which the hybrid dislocation passes. The loop expansion thus tests the use of the interpolation of core template corrections at angles in between the pre-computed angles.

Furthermore, in DDD, the segment lengths are adaptively selected based on the local dislocation curvature. High accuracy in problems with curvature at the atomistic scale requires segments lengths of $\sim 10|\boldsymbol{b}|$. Then, if a highly curved dislocation becomes very parallel to one face of the MD zone, applying a single core template across the entire pad thickness becomes less accurate. A typical challenging example is shown in Figure 8, where an accurate representation of the curved dislocation in the pad region is achieved only by using multiple character angles on successive segments. Such situations are handled naturally by the code, based on the automatic adaptation of segment lengths in DDD.

The dynamical evolution of the expanding hybrid loop is shown in Figure 9 via snapshots of the configuration at $0 \mathrm{ps}, 1 \mathrm{ps}, 4.5 \mathrm{ps}$ and $5.5 \mathrm{ps}$. The hybrid dislocation is indicated by the DDD nodes and the centro-symmetry parameter of the atoms. The detected DDD nodes in the atomistic region are also superimposed on the atomistic dislocation. The loop expands essentially equally in all directions, whether atomistic or DDD, and the shape preserves an overall symmetry despite different descriptions in the different domains. The 


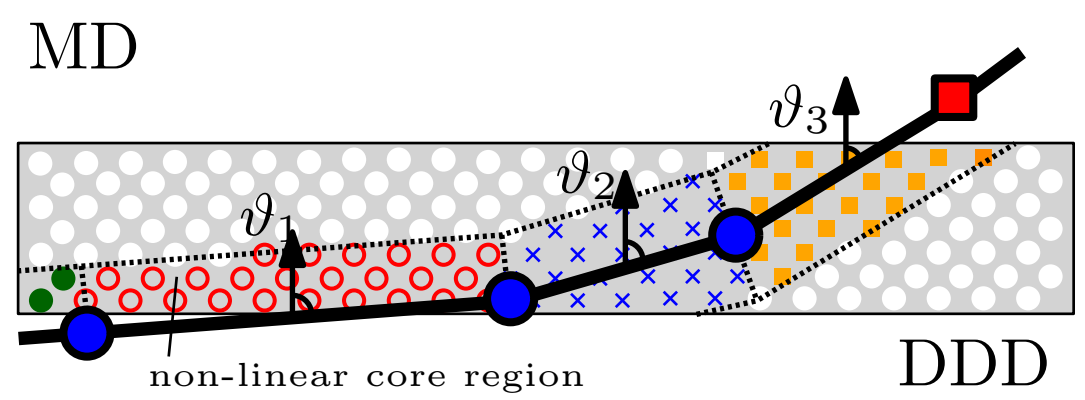

Figure 8: Application of several core templates with distinct character angles within the pad region. Around such a dislocation line, the pad region is decomposed into several sub-volumes each associated with a single DDD segment. On the figure three different angles $\left(\vartheta_{1}, \vartheta_{2}\right.$ and $\left.\vartheta_{3}\right)$ are applied to the red, blue and yellow atoms respectively.

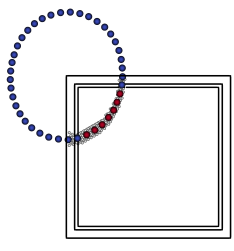

$0 p s$

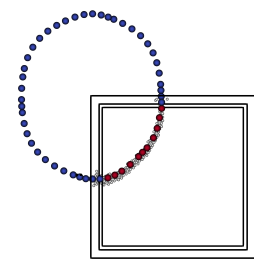

$1 p s$

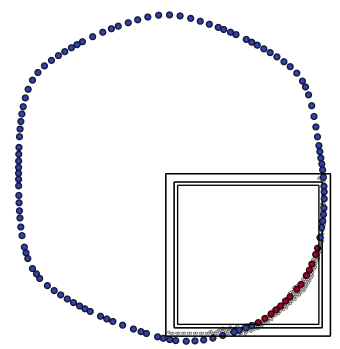

$4.5 p s$

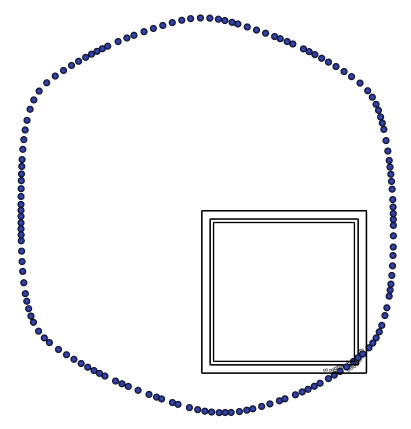

$5.5 p s$

Figure 9: The Hybrid dislocation loop at 0ps, 1ps, 4.5ps and 5.5ps. The DDD nodes are represented by the red circles. Inside of the atomic region, only the atoms violating centro-symmetry (with circles) as well as the DDD nodes (blue circles) are shown.

shape of the loop evolves due to the anisotropic mobility law, and the atomistic region shows a slightly more continuous, less polygonal, shape. This presumably reflects that the mobility laws for straight dislocations are not exactly accurate for describing highly curved dislocations. There are some small deviations in the configuration near the interface, but no unphysical behavior. CADD-3d smoothly handles the transitions around corners of the atomistic domain, where the local line character relative to the pad changes abruptly. Quantitatively, we have measured the dislocation positions where it intersects the dashed line indicated in Figure 7 that traverses the two domains. Figure 11 shows that the dislocation expands at essentially the same rate in both domains, consistent with the visual results of Figure 9.

CADD-3d can also capture more complex interacting dislocation arrangements. We demonstrate this capability by examining the expansion of the same loop but in the presence of two additional fixed elliptical loops residing in the continuum domain (Figure 10). Both loops have a long axis of $105|\boldsymbol{b}|$ and a short axis of $18|\boldsymbol{b}|$. The Burgers vectors of the fixed loops are set to be identical to that of the hybrid loop, which should generate repulsive forces on the hybrid loop. The forces acting on the atomistic portion of the hybrid dislocation are entirely captured through the displacements of the pad atoms due to the additional dislocations. The hybrid loop under the same shear load (400 MPa) after 5.5ps is shown in Figure 10b. As expected, as the hybrid dislocation expands and approaches the fixed DDD loops, the forces due to the fixed loops reduce the velocity along parts of the hybrid loop and the entire shape of the hybrid dislocation changes. The high symmetry of the overall loop is preserved, with the atomistic and continuum portions of the loop showing very similar behavior. The time evolution of the positions of the loop along the dashed line in Figure 7 are shown in Figure 11, showing good agreement between the motions of the two opposite parts of the hybrid dislocation during the entire time of expansion.

Finally, we examine the motion of the single hybrid dislocation loop under a shear load of only $70 \mathrm{MPa}$. Since the applied stress needed to expand the loop is $370 \mathrm{MPa}$, the loop should shrink and ultimately self-annihilate. As the loop contracts, the curvature increases and the distance between the two core templates (shown with the red hatching) decreases. When the two core templates overlap, CADD-3d simply superimposes the displacements, and this could generate spurious forces. Figure 12 shows the displacement 


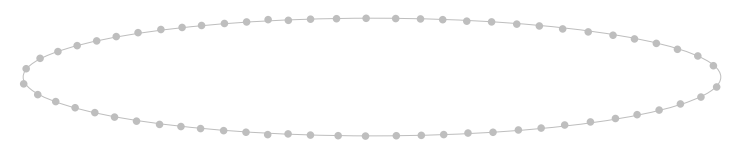



(a)

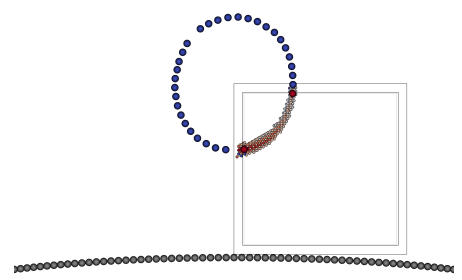

(b)

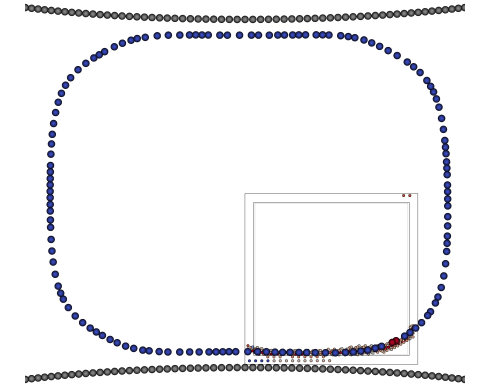

Figure 10: (a) Schematic of a hybrid dislocation loop placed between two fixed dislocation loops in the continuum domain; all Burgers vectors are equal. (b) Hybrid dislocation loop simulation at $\mathrm{t}=0 \mathrm{ps}$ and $\mathrm{ta}=5.5 \mathrm{ps}$ under a resolved shear stress of 400 MPa.

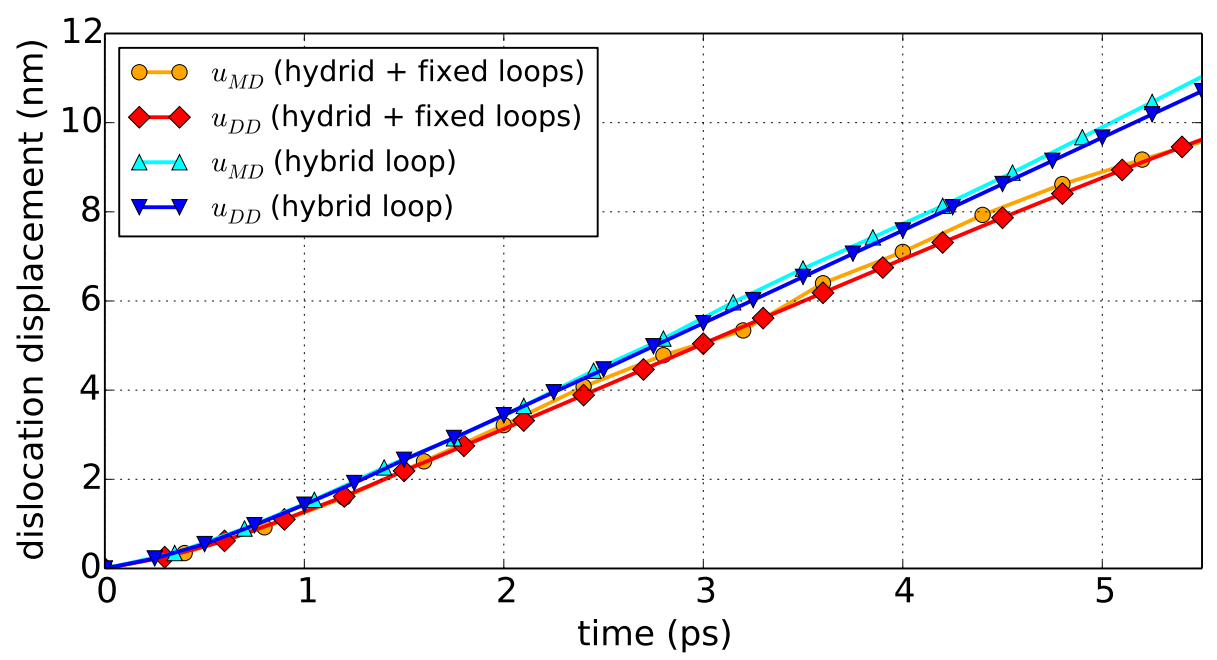

Figure 11: Position of the hybrid dislocation loop along the line shown in Figure 7 versus time, for both an isolated hybrid dislocation loop (Figure 7) and in the presence of two fixed DDD loops (Figure 10).

evolution of the MD and DDD sub-parts of the hybrid dislocation loop along the line $z-x=0$. At the beginning of the simulation, the two sub-parts shrink at a similar speed. As the loop curvature increases, the velocities start to deviate. However, the loop continues to shrink, the overlapping core templates do not halt the shrinkage, and the loop finally annihilates. The driving force for annihilation is high in this example, and this may overcome any spurious forces when the core templates overlap, but no deleterious features are observed in this case.

\section{Operation of a Frank-Read Source}

The operation of a Frank-Read (F-R) source is a canonical problem in dislocation mechanics, involving the evolution of an initial straight segment into a complex shape that eventually pinches off to create a complete loop and recreate the source, which can then operate again. Here, we examine the operation of a nanoscale double F-R using CADD-3d and compare the results to a fully atomistic simulation. We also compare to the fully DDD version of the same problem so as to highlight differences between DDD, full atomistics, and CADD-3d. To perform the fully atomistic simulation requires the use of a rather small-scale problem. Once we demonstrate the accuracy of CADD-3d, we will use it to examine a much larger scale.

At the atomistic level, it is necessary to study a complete dislocation loop and so the standard geometry is 


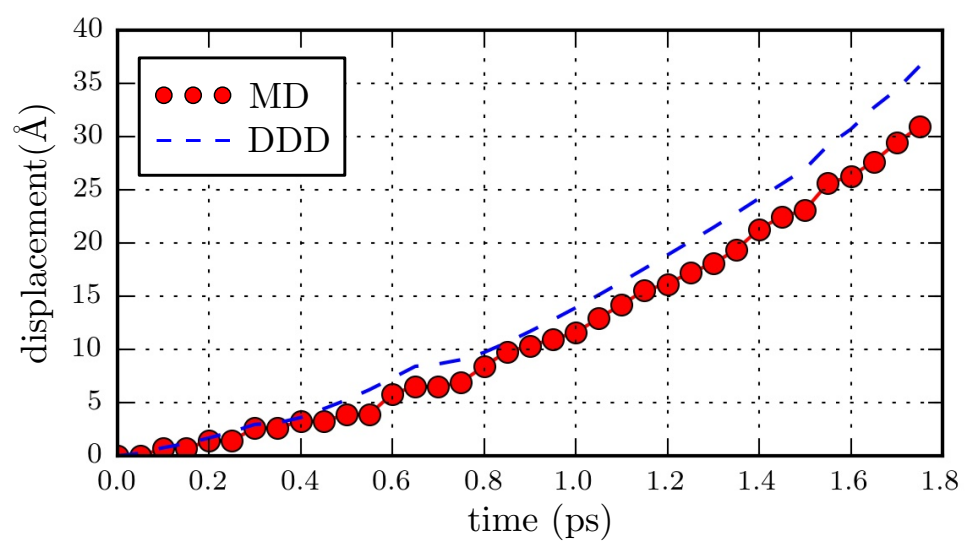

Figure 12: Contraction of the hybrid dislocation loop as measured by the positions of the MD and DDD portions of the loop along the dashed line indicated in Figure 7. The increasing deviation, while small, is attributed to a difference in mobility due to the increasing curvature.
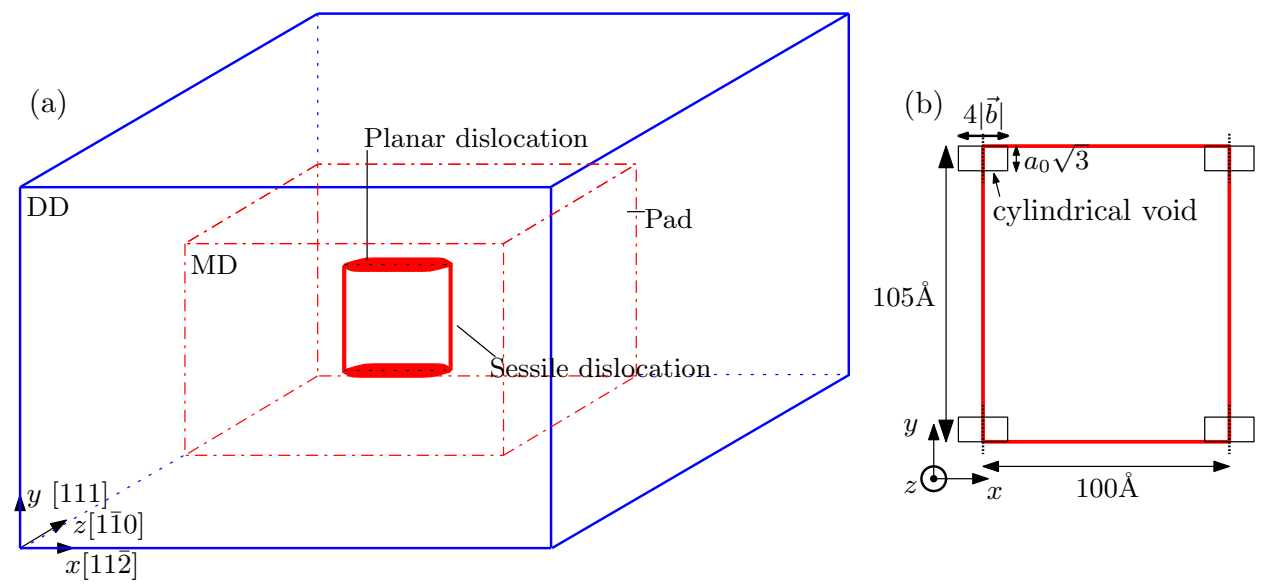

Figure 13: (a) Computational domain for the modeling of a double Frank-Read source in CADD-3d. The MD domain boundary is shown with the red solid lines, and the DDD boundary is indicated in the blue solid lines. (b) Details of the atomistic double F-R source, consisting of a loop formed by two glissile edge dislocations and two sessile dislocations. Nanoscale cylindrical voids are introduced at the corners of the loop to prevent motion of the atomistic sessile dislocations at the sharp corners.

the double F-R source, which consists of a rectangular loop with two sessile segments and two glide edge segments, as shown in Figure 13. Small voids are introduced at the corners of the loop to prevent motion of the sessile segments, as is described in [26]. For the small-scale study, the outer domain is a cube of lateral dimension size $L=57 \mathrm{~nm}$ and the atomistic domain has $\mathrm{x}-\mathrm{y}-\mathrm{z}$ dimensions $36 \mathrm{~nm} \times 21 \mathrm{~nm} \times 16 \mathrm{~nm}$. This domain is envisioned to be embedded inside an infinite continuum domain, but with all dislocations constrained to remain within the computational domain. Thus, the boundary conditions are as follows. For CADD-3d, the dislocation nodes are simply constrained to remain inside the computational domain (the mobility is set to zero when nodes reach the boundary). For MD, we fix the displacement fields of the atoms on the outer boundaries as if it was a pad region $(\hat{\boldsymbol{u}}+\tilde{\boldsymbol{u}})$ reacting to the motion of all dislocations. Without implementing the passing algorithm described in section 3 , the progression of the dislocations approaching the outer boundary will be stopped. The applied load is a uniform shear stress $\tau_{y z}$.

We consider a source with initial edge segments of length $10 \mathrm{~nm}$, and apply a stress $\tau_{y z}=700 \mathrm{MPa}$ sufficient to activate this small source. Figure 14 shows the evolution of the double F-R source system as a function of time, where only the dislocations emanating from the lower initial edge segment are shown. The dislocations from the upper initial edge segment behave similarly, but move in opposite directions. Figures $14 \mathrm{a}$, b, and c show the MD, CADD-3d, and DDD simulation results respectively. In all simulations, the initial source bows out, loops back on itself, pinches off to create a full loop and to recreate the source, which then operates again. The backstresses due to the previously emitted dislocations then inhibit the nucleation of subsequent loops. In CADD-3d, the initial fully-atomistic edge segment expands and partially passes into the DDD 
domain in good agreement with the full atomistic simulation. CADD-3d captures the annihilation/pinch-off process, even though this event takes place very close to the atomistic boundary, and regenerates the source, again in good agreement with the full atomistic simulation. The process repeats for the second loop, and then the source activity is suppressed. All configurations predicted by CADD-3d are in excellent agreement with the fully atomistic result. There are multiple dislocation passing events, continuously varying dislocation character at the atom/continuum interface, and complex topology changes, all captured well by CADD-3d.

There is a difference in the position of the second dislocation loop once equilibrium has been reached (i.e. when the source stops operating). This discrepancy is a consequence of the slightly different boundary conditions in CADD-3d and atomistics. Because of the small Peierls stress (10MPa), the position of a piling dislocation can vary substantially with the spurious stresses occurring near the atomistic boundary in the MD problem (see Paper II), however with very limited consequences onto the purely atomistic region. However, the difference in fields due to the different pile-up structures in MD and CADD-3d are dipolar in nature and thus short range, having limited influence on the source operation. There is also a small difference in the configuration of the final stable FR source segment, which is due to the small repulsive force of the elastic pad atoms in CADD-3d on the leading region of the loop, again related to the study shown in Paper II. Use of a larger MD domain (z dimension doubled, results not shown) reduces this error such that the final stable FR source segment is in excellent agreement with that found in the fully-atomistic study.

(a)



(b)

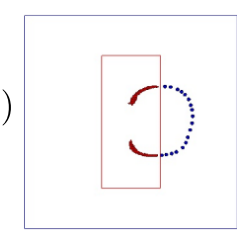

(c)

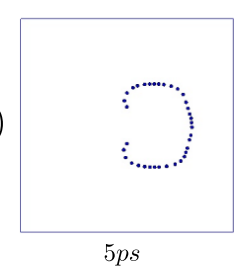

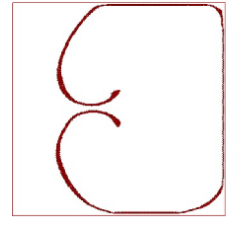
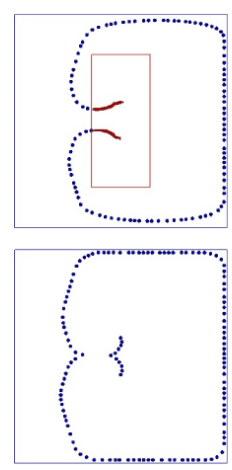

$10 p s$
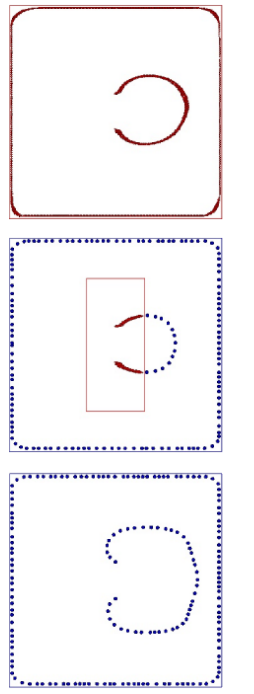

$17.5 p s$
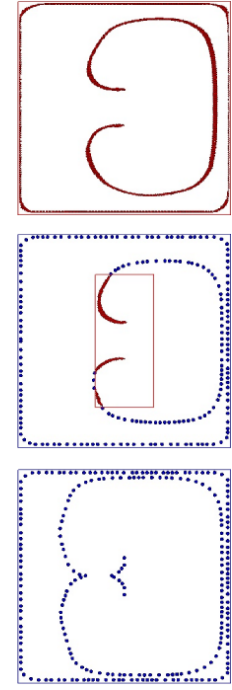

$20 \mathrm{ps}$
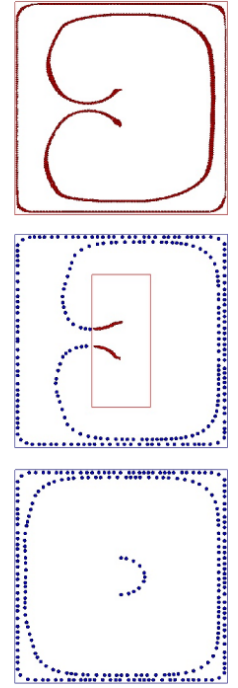

$25.5 p s$
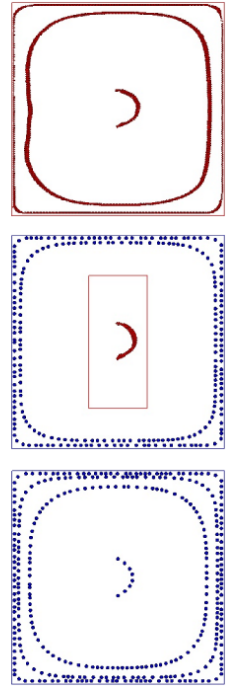

50 s

Figure 14: Time evolution of a double Frank-Read source under an applied shear, viewing only one source for clarity, as simulated in (a) full molecular dynamics, (b) CADD-3d, and (c) full discrete dislocation dynamics. MD and DDD domain boundaries are shown as red and blue solid lines, respectively. Red spheres: MD atoms in the dislocation core; blue spheres: DDD nodes.

In contrast to the good results for CADD-3d, the full DDD simulations deviate from atomistics. While showing the same general sequence of events, the DDD system evolves faster, has some differences in dislocation shape, and there is emission of a third dislocation before cessation of source operation. These differences may be attributable to the presence of the pinning voids, which create local fields and a drag at the surface intersected by the edge dislocation, and/or by the mobility/motion of the high-curvature atomistic dislocations for which the DDD mobility laws are not well-suited. These differences demonstrate, even in what would be considered a standard DDD problem, that atomistic effects at small scales do matter and cannot always be neglected. CADD-3d provides the ability to capture the atomistic phenomena with high precision while retaining the computational efficiency of DDD at larger scales where the DDD approach is sufficiently accurate.

With the successful validation of CADD-3d on a standard engineering plasticity problem at a scale accessible to full MD simulations, we now apply CADD-3d to the same double F-R source operation but at scales that are inaccessible by current MD. The geometry remains exactly that shown in Figure 13. The atomistic 
domain remains the same size as above but the outer DDD domain is expanded to $L=500 \mathrm{~nm}$, approximately eight times larger than the previous simulation. Since dislocations can glide out to the outer domain boundary, they exert smaller backstresses on the source and the source can thus operate many times, leading to the development of a dislocation pile-ups and significant long-range interactions between the source and the larger-scale plasticity. To the best of our knowledge, previous atomistic studies of FR sources [17, 26, 27] used small domains that lead to significant backstresses and the necessity of increasing the applied stress to continue source operation (strain hardening). Furthermore, previous DDD studies modeled FR sources in a simple way using pinned nodes $[6,2]$, which is not sufficient to capture atomistic effects at small scales appearing around the sessile dislocations (as seen in Figure 14).

In this study, we also model an $\mathrm{Al}-5 \% \mathrm{Mg}$ alloy, where the $\mathrm{Mg}$ atoms are randomly distributed in throughout the atomistic and pad domains. The interatomic potential for this case is the Ercolessi-Adams EAM potential for Al-Mg. The lattice constants and elastic constants are those for the alloy material $(\mu=48.8 G P a$ and $\nu=0.31$ ), the effective Peierls stress is adjusted to account for the solute strengthening of $\mathrm{Mg}$, and the core-template geometry is the same as pure $\mathrm{Al}$ aside from an expansion of the lattice constant. The mobility law was also adapted for the alloy. The interested reader can find more details in [8]. These factors are added to move beyond a simple study on a pure elemental fcc material and toward a realistic engineering alloy.

Figure 15a shows the entire dislocation network after 82 ps. Many dislocations have been nucleated from each edge segment, have passed into the continuum to form full loops, and generate an extended dislocation pile-up. A video of the full time evolution of the system can be found via the link given in [9]. Figure 15b shows the domain in and around the atoms, demonstrating the continuity of the evolving hybrid loops fully consistent with the results shown above.

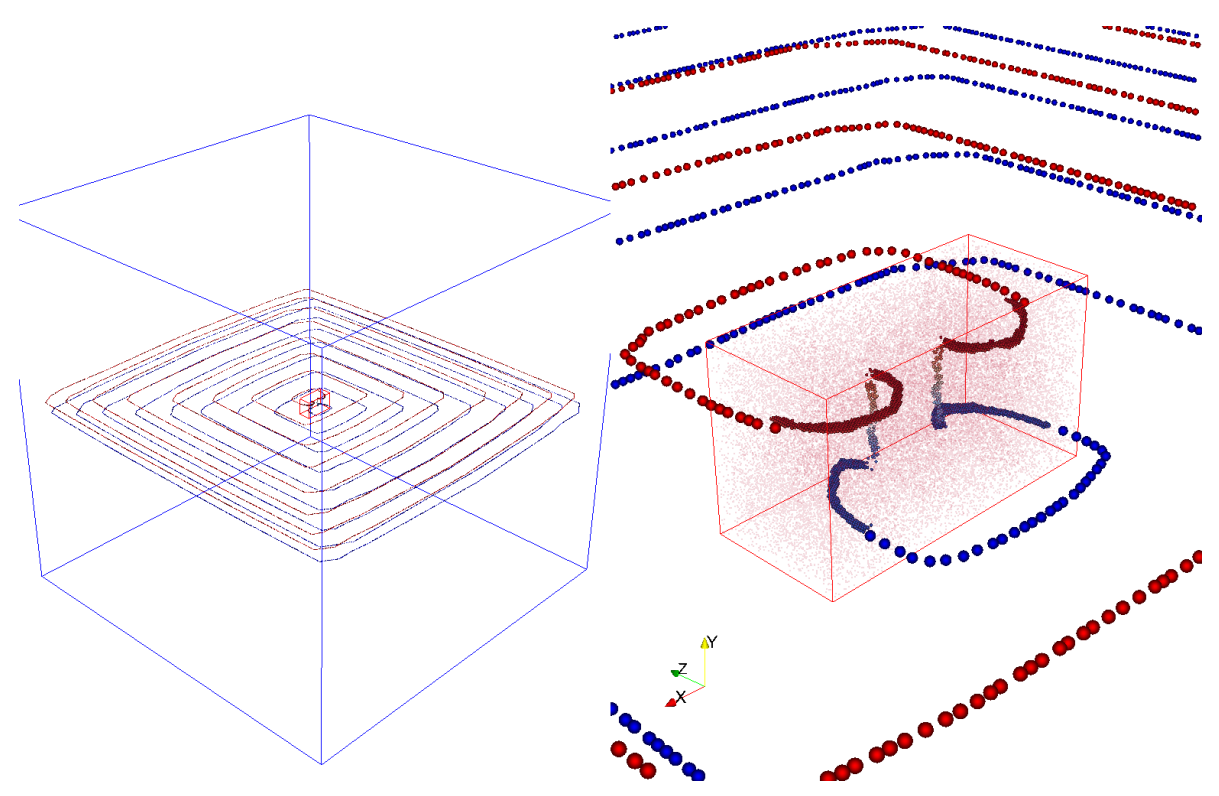

Figure 15: (a) Perspective view of dislocation loops nucleated from the atomistic FR source under a $1000 \mathrm{MPa}$ shear load after 82 ps. Red lines outline the MD cell. (b) Close-up view of the MD domain, where dislocated atoms and DDD nodes are colored by their $y$ coordinate. The red blur spheres indicate $\mathrm{Mg}$ atoms.

\section{Conclusion}

We have presented the application of CADD-3d to a sequence of increasingly complex time-dependent problems. Results for the motion of a single straight hybrid dislocation demonstrate the high fidelity of CADD-3d when the DDD parameters and core template are carefully calibrated. Results for expanding and shrinking hybrid dislocation loops show that CADD-3d can capture an evolving shape with evolving line character at the atomistic/continuum interface, with high curvatures, and with the same dynamics in both atomistic and continuum domains. Results further show that the hybrid dislocation evolves in response to the fields generated by additional dislocations in the overall network. Results for the activation of a double 
Frank-Read source demonstrate further fidelity with atomistic simulations, including loop expansion, passing from MD to DDD domains, pinching off, source reactivation, and source cessation. Finally, operation of the double F-R source at much larger scales $(500 \mathrm{~nm})$ in an metallic alloy demonstrates the power of CADD-3d for problems at the scales of engineering interest, where there are many interacting dislocations with a crucial underlying process that requires full atomistic resolution.

The sequence of Papers I, II, and III collectively present all the detailed algorithms of CADD-3d, the validation of key components of CADD-3d for static and dynamic reference problems, and the further validation and application of the method to complex dislocation plasticity problems. Full mechanical coupling of atoms to continuum was not used in this work, and implementation of that well-established aspect remains to be done $[16,23,30,15]$. We will report on progress toward efficient $3 \mathrm{~d}$ methods for the mechanical coupling in the near future. Nonetheless, the present CADD-3d sets the key foundation for future applications of the method in a variety of settings and provides the long-sought robust and highly accurate concurrent coupling of atomistic and dislocation plasticity.

\section{Acknowledgments}

The authors thank the Fonds National Suisse for support of this work through the project 200021_140506 entitled "Coupled Atomistic/Discrete-Dislocations in 3d (CADD-3d).

\section{Bibliography}

\section{References}

[1] Anciaux, G., Junge, T., Hodapp, M., Cho, J., Molinari, J.F., Curtin, W., . Coupled atomistic/discrete dislocation method in 3d - part i: Methoddology. Journal of the Mechanics and Physics of Solids .

[2] Arsenlis, A., Cai, W., Tang, M., Rhee, M., Oppelstrup, T., Hommes, G., Pierce, T.G., Bulatov, V.V., 2007. Enabling strain hardening simulations with dislocation dynamics. Modelling and Simulation in Materials Science and Engineering 15, 553.

[3] Barnett, D.M., 1985. The displacement field of a triangular dislocation loop. Philosophical Magazine A $51,383-387$.

[4] Barnett, D.M., Balluffi, R.W., 2007. The displacement field of a triangular dislocation loop-a correction with commentary. Philosophical Magazine Letters 87, 943-944.

[5] Bitzek, E., Gumbsch, P., 2004. Atomistic study of drag, surface and inertial effects on edge dislocations in face-centered cubic metals. Materials Science and Engineering: A 387, 11-15.

[6] Bulatov, V., Cai, W., 2006. Computer Simulations of Dislocations. Osmm Series, OUP Oxford.

[7] Cai, W., Bulatov, V.V., 2004. Mobility laws in dislocation dynamics simulations. Materials Science and Engineering: A 387-389, 277-281.

[8] Cho, J., 2017a. Coupled 3D Dislocation Modeling at Nano- and Micro-Scales. Ph.D. thesis. EPFL. Doi: 10.5075 /epfl-thesis-7598.

[9] Cho, J., 2017b. Video of a cadd3d frank-read source. URL: https://www.youtube.com/watch?v= jwK-TF7o20o.

[10] Cho, J., Junge, T., Molinari, J.F., Anciaux, G., 2015. Toward a 3d coupled atomistic and discrete dislocation dynamics simulation: dislocation core structures and Peierls stresses with several character angles in FCC aluminum. Advanced Modeling and Simulation in Engineering Sciences 2, 12.

[11] Cho, J., Molinari, J.F., Anciaux, G., 2017. Mobility law of dislocations with several character angles and temperatures in FCC aluminum. International Journal of Plasticity 90, 66-75. 
[12] Fivel, M., Depres, C., 2014. An easy implementation of displacement calculations in 3d discrete dislocation dynamics codes. Philosophical Magazine 94, 3206-3214.

[13] Hirth, J.P., Zbib, H.M., Lothe, J., 1998. Forces on high velocity dislocations. Modelling and Simulation in Materials Science and Engineering 6, 165. doi:10.1088/0965-0393/6/2/006.

[14] Hodapp, M., Anciaux, G., Molinari, J.F., Curtin, W., 2017. Coupled atomistic/discrete dislocation method in 3d - part ii: Validation of the method. Journal of the Mechanics and Physics of Solids .

[15] Junge, T., Anciaux, G., Molinari, J..F., 2015. Dynamic stability of displacement-based atomistic/continuum coupling methods. Journal of the Mechanics and Physics of Solids 80, $103-$ 120. URL: http://www.sciencedirect.com/science/article/pii/S002250961500071X, doi:https: //doi.org/10.1016/j.jmps.2015.04.004.

[16] Kohlhoff, S., Gumbsch, P., F., F.H., 1991. Crack propagation in b.c.c. crystals studied with a combined finite-element and atomistic model. Philosophical Magazine A 64, 851878. URL: https://doi.org/10.1080/01418619108213953, doi:10.1080/01418619108213953, arXiv:https://doi .org/10.1080/01418619108213953.

[17] de Koning, M., Cai, W., Bulatov, V.V., 2003. Anomalous Dislocation Multiplication in FCC Metals. Physical Review Letters 91.

[18] Mendelev, M., Kramer, M., Becker, C., Asta, M., 2008. Analysis of semi-empirical interatomic potentials appropriate for simulation of crystalline and liquid $\mathrm{Al}$ and $\mathrm{Cu}$. Philosophical Magazine 88, 1723-1750.

[19] Ni, L., Markenscoff, X., 2008. The self-force and effective mass of a generally accelerating dislocation I: Screw dislocation. Journal of the Mechanics and Physics of Solids 56, 1348-1379. doi:10.1016/j . jmps.2007.09.002.

[20] Olmsted, D.L., Jr, L.G.H., Curtin, W.A., Clifton, R.J., 2005. Atomistic simulations of dislocation mobility in $\mathrm{Al}, \mathrm{Ni}$ and $\mathrm{Al} / \mathrm{Mg}$ alloys. Modelling and Simulation in Materials Science and Engineering 13,371 .

[21] Pellegrini, Y.P., 2014. Equation of motion and subsonic-transonic transitions of rectilinear edge dislocations: A collective-variable approach. Physical Review B 90, 054120. doi:10.1103/PhysRevB.90. 054120.

[22] Pillon, L., Denoual, C., Pellegrini, Y.P., 2007. Equation of motion for dislocations with inertial effects. Physical Review B 76, 224105. doi:10.1103/PhysRevB.76.224105.

[23] Shenoy, V., Miller, R., Tadmor, E., Rodney, D., Phillips, R., Ortiz, M., 1999a. An adaptive finite element approach to atomic-scale mechanics - the quasicontinuum method. Journal of the Mechanics and Physics of Solids 47,611 - 642. URL: http://www.sciencedirect.com/science/article/pii/ S0022509698000519, doi:https://doi.org/10.1016/S0022-5096(98)00051-9.

[24] Shenoy, V.B., Miller, R., Tadmor, E.b., Rodney, D., Phillips, R., Ortiz, M., 1999b. An adaptive finite element approach to atomic-scale mechanics - the quasicontinuum method. Journal of the Mechanics and Physics of Solids 47, 611-642. doi:10.1016/S0022-5096(98)00051-9.

[25] Shilkrot, L., Miller, R.E., Curtin, W.A., 2004. Multiscale plasticity modeling: coupled atomistics and discrete dislocation mechanics. Journal of the Mechanics and Physics of Solids 52, 755-787.

[26] Shimokawa, T., Kitada, S., 2014. Dislocation Multiplication from the Frank-Read Source in Atomic Models. Materials Transactions 55, 58-63.

[27] Verdier, M., Fivel, M., Groma, I., 1998. Mesoscopic scale simulation of dislocation dynamics in fcc metals: Principles and applications. Modelling and Simulation in Materials Science and Engineering 6, $755-770$. 
[28] Wang, J., Lian, J., Greer, J.R., Nix, W.D., Kim, K.S., 2006. Size effect in contact compression of nanoand microscale pyramid structures. Acta Materialia 54, 3973-3982.

[29] Weygand, D., Friedman, L.H., Van der Giessen, E., Needleman, A., 2002. Aspects of boundary-value problem solutions with three-dimensional dislocation dynamics. Modelling and Simulation in Materials Science and Engineering 10, 437-468.

[30] Xiao, S., Belytschko, T., 2004. A bridging domain method for coupling continua with molecular dynamics. Computer Methods in Applied Mechanics and Engineering 193, 1645 - 1669. URL: http://www . sciencedirect.com/science/article/pii/S004578250400026X, doi:https://doi.org/10.1016/j . cma.2003.12.053. multiple Scale Methods for Nanoscale Mechanics and Materials. 
CADD-3d glossary
$\Omega_{\mathrm{A}} \quad$ Molecular dynamics region
$\Omega_{\mathrm{P}} \quad$ Boundary condition of the the Molecular dy- namics region. This is also referred as the pad region in the literature
$\partial \Omega_{\mathrm{I}} \quad$ Atomic region providing the boundary condi- tion to the linear elastic model. This region is often referred as the interface
$\Delta \tilde{\boldsymbol{u}}^{\text {corr }} \quad$ Core template correction field
$\Omega_{B} \quad$ Buffer zone employed for the dislocation pass- ing algorithm

\section{Atomistic glossary}

$r_{\mathrm{c}} \quad$ Inter-atomic cutoff radius

\section{Dislocation dynamics glossary}

\begin{tabular}{|c|c|}
\hline & $\begin{array}{l}\text { eld computed with infinite boundary condi- } \\
\text { ns following [29] }\end{array}$ \\
\hline & $\begin{array}{l}\text { eld computed from an elastic boundary } \\
\text { lue problem following [29] }\end{array}$ \\
\hline core & $\begin{array}{l}\text { pre Radius which is characterizing the non } \\
\text { ear region around the dislocation line }\end{array}$ \\
\hline & $\begin{array}{l}\text { Dislocation segments with at least one end } \\
\text { located in the continuum region }\end{array}$ \\
\hline & $\begin{array}{l}\text { Virtual dislocation segments employed to } \\
\text { close the loops on } \partial \Omega_{\mathrm{I}}\end{array}$ \\
\hline$i$ & $\begin{array}{l}\text { ement field due to the presence of dis- } \\
\text { as within infinite boundary conditions }\end{array}$ \\
\hline & Burgers vector \\
\hline$m^{d}$ & $\begin{array}{l}\text { Dislocation effective mass employed in th } \\
\text { modified mobility law }\end{array}$ \\
\hline & Resolved shear stress \\
\hline & Peierls stress \\
\hline
\end{tabular}

\section{Continuum elasticity}

$\hat{\boldsymbol{u}}$ Dislocation free displacement field

$\boldsymbol{u}$ Displacement field

$\hat{\boldsymbol{\sigma}}$ Dislocation free stress tensor field

$\mu$ Shear modulus (approximation for aluminum) 